\title{
Preoperative serum albumin: a promising indicator of early mortality after surgery for infective endocarditis
}

\author{
Suiqing Huang ${ }^{1,2 \#}$, Zhuoming Zhou ${ }^{1,2 \#}$, Li Luo ${ }^{1,2 \#}$, Yuan Yue ${ }^{1,2}$, Quan Liu ${ }^{1,2}$, Kangni Feng ${ }^{1}$, Jian Hou ${ }^{1,2}$, \\ Keke Wang ${ }^{3}$, Jiantao Chen ${ }^{1}$, Huayang $\mathrm{Li}^{1,2}$, Lin Huang ${ }^{1,2}$, Guangguo Fu ${ }^{1,2}$, Guangxian Chen ${ }^{1}$, Mengya Liang ${ }^{1}$, \\ Zhongkai $\mathbf{W u}^{1}$
}

${ }^{1}$ Department of Cardiac Surgery, First Affiliated Hospital of Sun Yat-sen University, Guangzhou, China; ${ }^{2}$ NHC Key Laboratory of Assisted Circulation, Sun Yat-sen University, Guangzhou, China; ${ }^{3}$ Department of Emergency, First Affiliated Hospital of Sun Yat-sen University, Guangzhou, China Contributions: (I) Conception and design: S Huang, Z Zhou; (II) Administrative support: Z Wu; (III) Provision of study materials or patients: Z Wu, M Liang; (IV) Collection and assembly of data: S Huang, Z Zhou, L Luo, Y Yue, Q Liu, K Feng, J Hou, K Wang; (V) Data analysis and interpretation: L Luo, J Chen, H Li, L Huang, G Fu, G Chen; (VI) Manuscript writing: All authors; (VII) Final approval of manuscript: All authors.

"These authors contributed equally to this work.

Correspondence to: Zhongkai Wu, MD, PhD; Mengya Liang, MD. Department of Cardiac Surgery, First Affiliated Hospital of Sun Yat-sen University, 58 Zhongshan II Rd, Guangzhou 510080, China. Email: wuzhk@mail.sysu.edu.cn; infisdsums@163.com.

Background: Despite novel improvements in the diagnosis and treatment of infective endocarditis (IE), there has been no significant improvement in the survival rate of IE, which indicates that many details still need to be optimized in the preoperative assessment. We sought to evaluate preoperative serum albumin as a biomarker for predicting early mortality after IE surgery.

Methods: Between October 2013 and June 2019, patients with a definite diagnosis of IE were enrolled in this study. Patients' albumin levels at admission were used as the preoperative albumin levels. Restricted cubic spline and multivariate logistic regression analyses were performed to evaluate the relationship between albumin and early mortality. Receiver operating characteristic curve analyses were performed to assess the role of albumin in predicting early mortality and compare the predictive capacity of traditional models with models that included albumin.

Results: Of the 276 IE patients, 20 (7.2\%) died in hospital or within 30 days of surgery. Hypoalbuminemia (an albumin level <3.5 g/dL) was present in 109 (39.5\%) patients. The multivariate logistic regression analysis showed that preoperative albumin was inversely associated with early mortality [adjusted odds ratio $(\mathrm{OR})=0.22$ per $1 \mathrm{~g} / \mathrm{dL}, 95 \%$ confidence interval $(\mathrm{CI}): 0.07-0.65, \mathrm{P}=0.006]$ after full adjustment. Preoperative albumin had value in predicting early mortality [area under the curve (AUC) $=0.72,95 \%$ CI: 0.61-0.84; $\mathrm{P}<0.01]$. After adding albumin to the European System for Cardiac Operative Risk Evaluation (EuroSCORE) and Charlson score, the predictive ability of the model was further improved (EuroSCORE II: AUC $=0.55$; 95\% CI: $0.42-0.67$ to AUC $=0.72 ; 95 \%$ CI: 0.61-0.84; Charlson score: AUC $=0.73 ; 95 \%$ CI: $0.64-0.83$ to $\mathrm{AUC}=0.78 ; 95 \% \mathrm{CI}: 0.68-0.88)$.

Conclusions: Preoperative serum albumin is inversely associated with early mortality after IE surgery, and is a promising prognostic indicator in preoperative risk stratification assessments of IE patients.

Keywords: Albumin, infective endocarditis (IE); cardiac surgery; early prognosis

Submitted Jul 19, 2021. Accepted for publication Sep 03, 2021.

doi: $10.21037 /$ atm-21-3913

View this article at: https://dx.doi.org/10.21037/atm-21-3913 


\section{Introduction}

Infective endocarditis (IE) is an infection of the cardiac endothelium, such as the heart valve, chordae tendineae, interventricular septum, mural endocardium, or large intrathoracic vessels. Despite novel improvements in the diagnosis and treatment of IE, both the incidence and mortality rates of the disease have remained high over the years $(1,2)$. In developed countries, IE has an in-hospital mortality rate of around $20 \%$, which increases to $40 \%$ at 1 year (3). The condition of IE is complex, and involves infections, cardiovascular system disorders, and even complications of other systems (e.g., acute renal failure, neurological complication, and splenic complication). Extensive evidence indicates that in IE, surgical treatment leads to a better prognosis than conservative treatment (4). However, for patients who require surgery, preoperative evaluations are intricate. Several studies have identified numerous risk factors for the prognosis of IE, including age, urgent or emergency surgery, multivalvular affection, renal failure, and staph aureus infection (5). However, in the past 2 decades, there has been no significant improvement in the survival rate of IE (6), which indicates that many details still need to be optimized in the preoperative assessment and management of IE.

Albumin is a $69 \mathrm{kDa}$ protein, which accounts for about $60 \%$ of the total plasma protein pool (7). For a long time, hypoalbuminemia has been a powerful prognostic marker in the general population and many pathological settings. Recent guidelines recommend that patients with low preoperative serum albumin levels $\left(<3.0 \mathrm{~g} \cdot \mathrm{dL}^{-1}\right)$ should avoid surgery, as such levels are associated with an impaired nutritional status and poor outcomes (8). Hypoalbuminemia is related to the appearance of various cardiovascular diseases and has become a powerful prognostic parameter for patients with cardiovascular diseases undergoing surgeries, including a coronary artery bypass graft, valve surgery, aortic surgery, or combined surgery $(7,9)$.

Malnutrition is an important factor that regulates albumin production; however, serum albumin is also affected by various non-nutritional factors, such as chronic inflammation, repeated infections, liver failure, renal insufficiency, gastrointestinal function changes, and fluid overload dilution (10). Studies have shown that albumin is also decreased in IE patients (11), and the cause is not only malnutrition but also inflammation, infection, and renal insufficiency. Among these factors, severe sepsis, malnutrition and acute kidney injury are also high-risk factors for the morbidity and mortality of IE (12-15). Preoperative hypoalbuminemia represents the sum of different factors associated with poor surgical outcomes in IE. In addition, experimental and clinical evidence indicates that hypoalbuminemia promotes pulmonary edema, myocardial edema, and water and sodium retention, which are related to adverse outcomes in patients who have been diagnosed with or are at risk of heart failure (16). It also robs the body of the albumin's antioxidant, antiinflammatory, and anti-platelet aggregation functions (7), thereby exacerbating disease progression. Therefore, preoperative low albumin reflects its predictive value for poor prognosis in IE from many aspects. First of all, the causes of hypoalbuminemia are important factors in the poor prognosis of IE surgery patients. Additionally, hypoalbuminemia further aggravates the severity of the disease.

This study sought to demonstrate the association between preoperative serum albumin and postoperative early mortality after IE surgery, and to evaluate the prognostic value of preoperative serum albumin to further understand its role in the preoperative risk stratification assessment of IE patients. Compared with previous studies, we analyzed and discussed the relationship between preoperative serum albumin and the early prognosis of IE for the first time. And we added serum albumin to the traditional risk assessment scoring system to discuss its effect on the prognostic ability of the model.

We present the following article in accordance with the STARD reporting checklist (available at https://dx.doi. org/10.21037/atm-21-3913).

\section{Methods}

\section{Study population and data extraction}

This study was conducted according to the Declaration of Helsinki (as revised in 2013) and was approved by the Institutional Review Board of The First Affiliated Hospital of Sun Yat-sen University (IRB-FAH-SYSU). Individual consent for this retrospective analysis was waived. We reviewed our database of patients from October 2013 to June 2019 and 441 consecutive patients who met definite modified Duke criteria for infective endocarditis at The First Affiliated Hospital of Sun Yat-sen University were identified. We excluded patients who had not undergone heart surgery, were pregnant, had life threatening malignancy, were participating in clinical trials or missed 
Participants approached for screening

$$
(n=441)
$$

- Consecutive patients admitted to our hospital between October 2013 and June 2019 who met definite modified Duke criteria for infective endocarditis

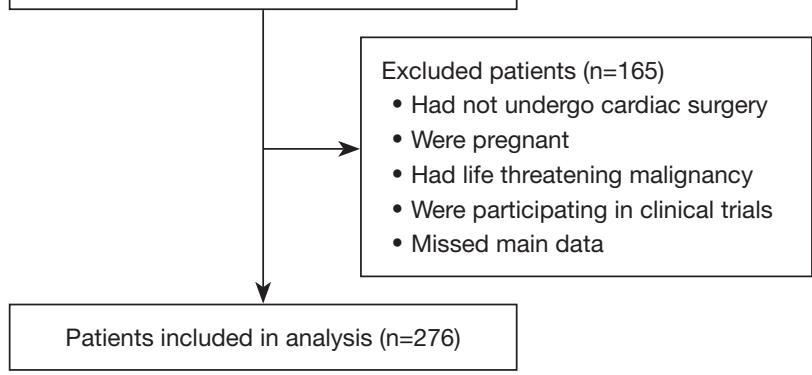

Figure 1 Patient flowchart.

main data. Ultimately, a total of 276 patients were included in the study population (see Figure 1).

Participants for surgical treatment were identified in accordance with the European Society of Cardiology Guidelines for the management of IE. Data were obtained from patients' electronic medical records using the information system of The First Affiliated Hospital of Sun Yat-sen University and the intensive care medical record system of The First Affiliated Hospital of Sun Yat-sen University. The extracted data included: (I) demographic information; (II) medical history; (III) echocardiography and laboratory events; (IV) IE-related characteristics; (V) operative characteristics; and (VI) European System for Cardiac Operative Risk Evaluation (EuroSCORE II) and Charlson scores.

\section{Outcome and variable definitions}

The primary outcome of our study was all-cause early mortality, which included mortality that occurred in hospital and within 30 days of the surgical procedure. Patients' albumin levels at admission were used as the preoperative albumin levels, and hypoalbuminemia was defined as an admission albumin $<3.5 \mathrm{~g} / \mathrm{dL}$. Heart failure was defined as New York Heart Association class III/IV. Renal insufficiency was defined as a glomerular filtration rate $<60 \mathrm{~mL} / \mathrm{min} /$ $1.73 \mathrm{~m}^{2}$. Other comorbidities were defined according to the International Classification of Diseases, Ninth Revision, Clinical Modification codes. All the performers of the test were blinded to all patient information.

\section{Statistical analysis}

Continuous variables are presented as the mean \pm standard deviation (SD), and categorical data are presented as numbers with proportions. Logistic regression models were applied for the univariate and multivariate analyses to identify the association between preoperative albumin and early mortality. Model 1 was adjusted for the EuroSCORE II; Model 2 was adjusted for the Charlson score; and Model 3 was adjusted for the key risk factors, including age, heart failure, renal insufficiency, multiple valve infection, and vegetation size. The results are presented as odds ratios (ORs) and 95\% confidence intervals (CIs). The visualized relationship between preoperative albumin and risk of hospital mortality was represented by a restricted cubic spline (RCS) curve with knots at the 5th, 50th, and 95th percentiles of its distribution, and adjusted with covariates in Model 3. Receiver operating characteristic (ROC) curves were constructed, and area under the curve (AUC), sensitivity, and specificity were calculated to compare the predictive capability of preoperative albumin with the EuroSCORE II and the Charlson score for early mortality. All tests were two-sided, and $\mathrm{P}$ values less than 0.05 were considered significant. Indeterminate results were considered false-positive or false-negative and incorporated into the final analysis. Missing data were handled by exclusion of these vessels as well as by the worst-case imputation. All statistical analyses were performed using $\mathrm{R}$, version 3.6.3 (R Foundation for Statistical Computing, http://www.R-project.org) and STATA, version 14.0 (StataCorp, College Station, TX, USA).

\section{Results}

\section{Patient characteristics}

A total of 276 patients who met the selection criteria were enrolled in the study, and a total of 20 deaths (7.2\%) occurred in hospital or within 30 days of the surgical procedure. The baseline characteristics of the patients are briefly summarized in Table 1 . The mean age of patients was $43.7 \pm 15.3$ years; $82(29.7 \%)$ patients were female. The mean preoperative albumin value was $3.6 \pm 0.6 \mathrm{~g} / \mathrm{dL}$. Hypoalbuminemia (admission albumin level $<3.5 \mathrm{~g} / \mathrm{dL}$ ) was present in 109 (39.5\%) patients. The mean time between albumin examination and outcome was 40.1 days (range, 


\section{Page 4 of 9}

Table 1 Baseline characteristics of surgically treated infective endocarditis patients

\begin{tabular}{|c|c|}
\hline Variables & Overall population $(n=276)$ \\
\hline \multicolumn{2}{|l|}{ Demographics } \\
\hline Age, years & $43.7 \pm 15.3$ \\
\hline Female, n (\%) & $82(29.7)$ \\
\hline \multicolumn{2}{|l|}{ Medical history, n (\%) } \\
\hline Hypertension & $40(14.5)$ \\
\hline Coronary artery disease & $13(4.7)$ \\
\hline Diabetes mellitus & $10(3.6)$ \\
\hline Atrial fibrillation & $23(8.3)$ \\
\hline Congenital heart disease & $53(19.2)$ \\
\hline Cerebral infarction & $58(21.0)$ \\
\hline Previous cardiac surgery & $17(6.2)$ \\
\hline Heart failure & $47(17.0)$ \\
\hline Current smoker & $37(13.4)$ \\
\hline Moderate to severe anemia & $46(16.7)$ \\
\hline Renal insufficiency & $17(6.2)$ \\
\hline \multicolumn{2}{|l|}{ Echocardiography and laboratory } \\
\hline LVEF, \% & $66.8 \pm 8.4$ \\
\hline Pulmonary arterial pressure, $\mathrm{mmHg}$ & $40.5 \pm 13.6$ \\
\hline Preoperative albumin, $\mathrm{g} / \mathrm{dL}$ & $3.6 \pm 0.6$ \\
\hline Hypoalbuminemia, n (\%) & 109 (39.5) \\
\hline \multicolumn{2}{|l|}{ IE-related characteristics } \\
\hline \multicolumn{2}{|l|}{ Affected valves, n (\%) } \\
\hline Aortic valve & $85(30.8)$ \\
\hline Mitral valve & $109(39.5)$ \\
\hline Tricuspid valve & $11(4.0)$ \\
\hline Aortic and mitral valve & $46(16.7)$ \\
\hline Multiple valve & $52(18.8)$ \\
\hline Prosthetic valve & $9(3.3)$ \\
\hline Perivalvular abscess, n (\%) & $23(8.3)$ \\
\hline Vegetation size, $\mathrm{mm}$ & $11.2 \pm 7.1$ \\
\hline \multicolumn{2}{|l|}{ Operative characteristics } \\
\hline \multicolumn{2}{|l|}{ Surgical procedure, n (\%) } \\
\hline Valve replacement & $261(94.6)$ \\
\hline Valve repair & $15(5.4)$ \\
\hline Concomitant CABG & $3(1.1)$ \\
\hline Concomitant aortic surgery & $9(3.3)$ \\
\hline Other procedures & $39(14.1)$ \\
\hline
\end{tabular}

Table 1 (continued)
Huang et al. Albumin: an early surgical prognostic marker of IE

Table 1 (continued)

\begin{tabular}{lc}
\hline Variables & Overall population $(\mathrm{n}=276)$ \\
\hline Scores & \\
EuroSCORE II & $2.9 \pm 2.4$ \\
Charlson score & $1.7 \pm 1.6$ \\
\hline
\end{tabular}

Values are presented as the mean \pm standard deviation for continuous variables, or the number of patients (\%) for categorical variables. EuroSCORE, European System for Cardiac Operative Risk Evaluation; LVEF, left ventricle ejection fraction; CABG, coronary artery bypass graft .

13-145 days). The mean score of patients was $2.9 \pm 2.4$ and $1.7 \pm 1.6$ for the EuroSCORE II and Charlson score, respectively. No significant adverse events occurred as a result of testing.

\section{Relationship between preoperative albumin and early mortality}

Both the univariate and multivariate analyses showed an inverse association between preoperative albumin and the risk of early mortality. When adjusted with the EuroSCORE II or Charlson score, the ORs for preoperative albumin were 0.23 (95\% CI: $0.10-0.54 ; \mathrm{P}=0.001)$ and $0.25(95 \%$ CI: $0.10-0.62 ; \mathrm{P}=0.003)$ for early mortality, respectively. After full adjustment, the admission albumin level remained inversely associated with early mortality (adjusted OR $=0.22$ per $1 \mathrm{~g} / \mathrm{dL}, 95 \%$ CI: $0.07-0.65 ; \mathrm{P}=0.006$ ) (see Table 2 ). After a similar adjustment, hypoalbuminemia (albumin $<3.5 \mathrm{~g} / \mathrm{dL}$ ) was found to be consistently associated with higher early mortality (adjusted OR 3.09, 95\% CI: $1.02-$ 9.35; $\mathrm{P}=0.046$ ) (see Table S1). The RCS adjusted with covariates further confirmed that preoperative albumin was inversely associated with the risk of early mortality and revealed a linear relationship (see Figure 2).

\section{Prognostic predictive ability of albumin}

To assess the potential predictive role of preoperative albumin in early mortality, and to compare the predictive capacity of traditional models with models that included preoperative albumin, ROC curve analyses was performed. The AUC for albumin only was 0.72 (95\% CI: 0.61-0.84). Table S2 shows preoperative hypoalbuminemia in relation to prediction of early mortality. EuroSCORE II alone was a poor predictor of early mortality (AUC $=0.55 ; 95 \%$ CI: $0.42-0.67)$; however, adding preoperative albumin to 
Table 2 Univariate and multivariate logistic regression analyses showing the association between preoperative albumin and the risk of early mortality in surgically treated infective endocarditis patients

\begin{tabular}{lcc}
\hline & Odds ratio (95\% Cl) & P value \\
\hline Univariate analysis & & \\
Albumin, per $1 \mathrm{~g} / \mathrm{dL}$ & $0.23(0.10-0.54)$ & 0.001 \\
Multivariate analysis & & \\
Model 1 & $0.23(0.10-0.54)$ & 0.001 \\
Albumin, per 1 g/dL & $0.97(0.78-1.19)$ & 0.749 \\
EuroScoRE II & & \\
Model 2 & $0.25(0.10-0.62)$ & 0.003 \\
Albumin, per 1 g/dL & $1.46(1.11-1.92)$ & 0.007 \\
Charlson score & & \\
Model 3 & $0.22(0.07-0.65)$ & 0.006 \\
Albumin, per 1 g/dL & $1.00(0.97-1.04)$ & 0.798 \\
Age, per year & $7.52(2.68-21.12)$ & $<0.001$ \\
Heart failure & $2.94(0.63-13.67)$ & 0.168 \\
Renal insufficiency & $4.35(1.46-12.96)$ & 0.008 \\
Multiple valve infection & $0.96(0.89-1.04)$ & 0.326 \\
Vegetation size, per 1 mm & & \\
\hline
\end{tabular}

EuroSCORE, European System for Cardiac Operative Risk Evaluation.

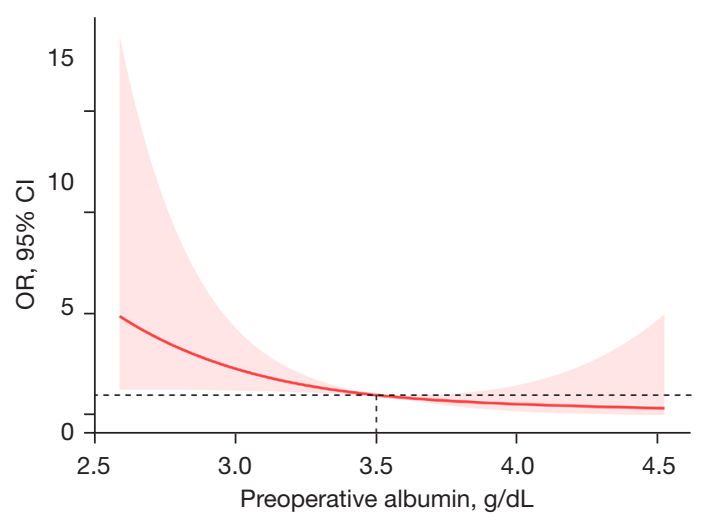

Figure 2 Restricted cubic spline demonstrating the relationship between the preoperative albumin and adjusted odds ratio (with a $95 \%$ confidence interval) of early mortality in surgically treated infective endocarditis patients.

the EuroSCORE II significantly improved the predictive ability of the model (AUC $=0.72$; 95\% CI: $0.61-0.84$; P for model improvement: <0.001). The AUC of the Charlson score alone was 0.73 (95\% CI: 0.64-0.83). After adding preoperative albumin to the Charlson score, the predictive ability of the model was improved, but the difference was not statistically significance (AUC $=0.78$; 95\% CI: 0.68 0.88; P for model improvement: 0.069) (see Figure 3).

\section{Discussion}

Due to the high fatality rate and complexity of the preoperative condition of IE, many studies have been conducted on patient characteristics to explore their impact on poor outcomes. A meta-analysis of 16 studies with a total of 7,484 patients showed that many characteristics and complications were important risk factors for early postoperative death, including age, female sex, urgent or emergency surgery, previous cardiac surgery, NYHA $\geq \mathrm{III}$, cardiogenic shock, prosthetic valve, multivalvular affection, renal failure, paravalvular abscess and S. aureus infection (5). Research on risk factors helps us grasp the timing of surgery. Appropriate early surgery can prevent serious complications or even death, and the timing of surgical intervention should be determined by a multidisciplinary team of cardiologists, cardiac surgeons, and infectious disease specialists. Heart failure, uncontrollable infection, and embolism prevention are the most common reasons for patients to undergo early surgery. However, patients with large cerebral infarction or intracerebral hemorrhage should defer surgery, as early surgery carries a significant risk of neurological deterioration and perioperative intracerebral hemorrhage. Decisions about the timing of surgery should weigh the individual's risks and benefits, and if the benefits outweigh the risks of surgery, surgery should be performed as early as possible (17). This study aims to further discuss the value of serum albumin in the preoperative evaluation of infective endocarditis. Our study found that the incidence of hypoalbuminemia in patients with IE was high, and that preoperative serum albumin is an independent risk factor and a potential prognostic predictor of early mortality after IE surgery.

Currently, there is no direct evidence that elucidates the mechanism by which hypoalbuminemia serves as a risk factor for postoperative early mortality in IE surgery. First, from the perspective of etiology, IE is often accompanied by severe infections and even sepsis. The systemic inflammatory response causes extensive endothelial damage, leading to microcapillary leakage, which is the main mechanism by which plasma protein flows through capillaries into the extravascular space, causing hypoalbuminemia (18). Second, 

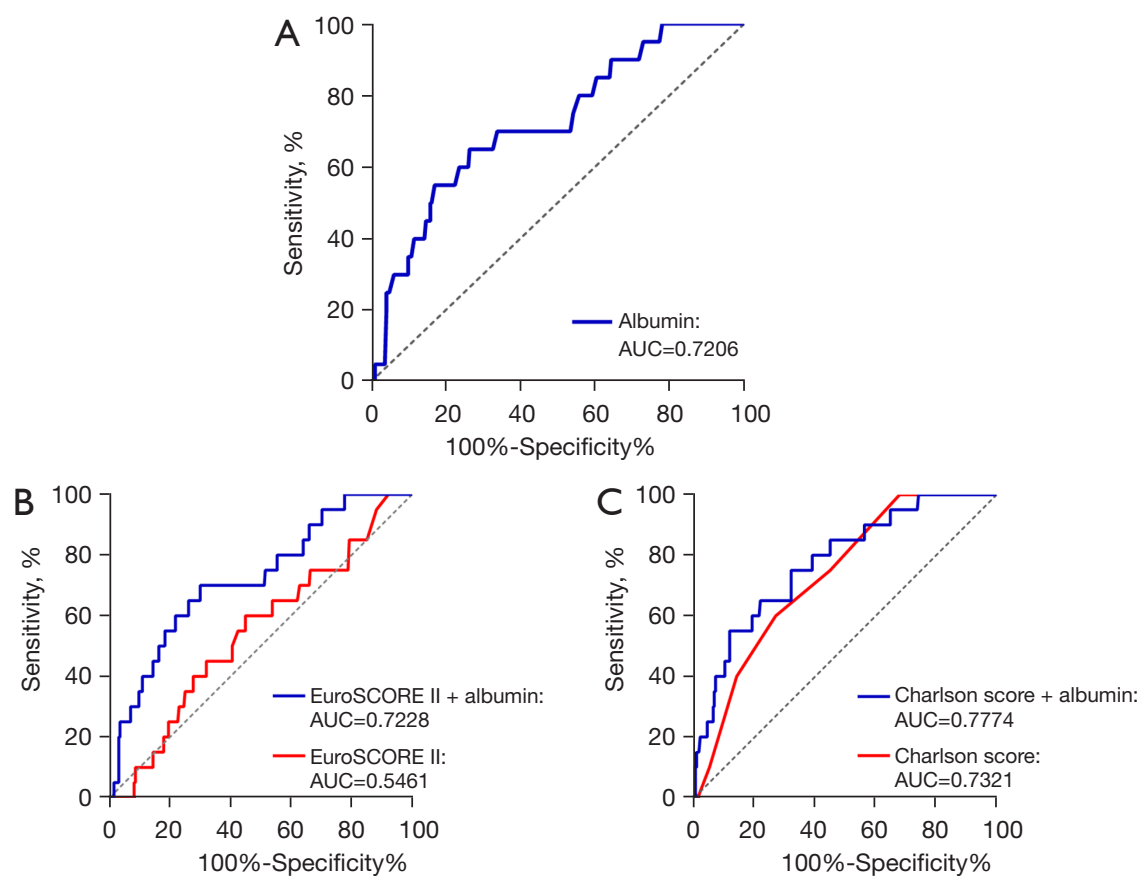

Figure 3 ROC curves comparing the capability of preoperative albumin in different models in predicting early mortality. (A) The predictive value of albumin only; (B) albumin adds additional predictive value to the EuroSCORE II. (C) Albumin adds additional predictive value to the Charlson score. AUC, area under the curve; EuroSCORE, European System for Cardiac Operative Risk Evaluation; ROC, receiver operating characteristic.

infection and heart failure in IE are accompanied by the excessive activation of the body's immune system, a loss of appetite and dietary restrictions in patients, which often lead to the consumption of nutrients and the further reduction of albumin (19). Finally, acute renal failure occurs in about $6-30 \%$ of IE patients and is a common complication in this population, which results in the loss of albumin from urine $(2,14)$. These causes are also risk factors of in-hospital death and postoperative adverse cardiovascular events in IE patients $(12-15,20)$.

Conversely, from the perspective of pathophysiological function, albumin may have a protective effect on inflammation and oxidative stress-related endothelial dysfunction (21), and the normal function of the immune response system depends on metabolism and nutritional status (19). Higher albumin is associated with nutrient reserves and a more efficient metabolic state, which can help ward off inflammation and immune responses to surgery (8). However, albumin helps to maintain the stability of the capillary membrane and the fluid balance across the capillary wall through its colloid osmotic effect and interaction with the endothelial glycocalyx.
Hypoalbuminemia leads to net extravasation of fluid into the interstitial spaces, thereby promoting pulmonary edema, myocardial edema, and fluid retention, which ultimately aggravate heart failure (16). In addition, some studies have shown that albumin may play a protective role in myocardial damage by acting as a natural inhibitor of the angiotensin-converting enzyme (22). In conclusion, a deficiency of albumin leads to the loss of anti-inflammatory and antioxidant functions and the aggravation of heart failure in IE patients. Additionally, following the onset and progression of the disease, a large amount of albumin is consumed. Hypoproteinemia, a vicious-cycle factor, further aggravates the disease and worsens the prognosis of IE patients. Research by Serkan Kahraman el. indicates that the prognostic nutritional index, based on serum albumin and lymphocyte concentration, is a nutritional score based on inflammation and considered to be a poor prognostic indicator for IE patients (23). Consistent with the current study, the main mechanisms include that albumin is a good indicator of nutritional status in addition to its influence on osmotic pressure and has antioxidant and anti-inflammatory properties. However, the study differs from our study in 
that prognostic nutritional index also involved lymphocyte number, focused more on inflammatory response. And lymphocytopenia and hypoalbuminemia are independent risk factors for acute kidney injury, which is also likely mechanism for poor prognosis in IE. Some studies have also examined molecular markers in patients with IE to explore their prognostic value. Siciliano et al. measured nine biomarkers in IE patients at admission and on day 7 of antibiotic treatment and found that s-c TnI had the highest early prognostic value (24). However, unlike this study, which included both surgical and non-surgical patients, our study only included surgical patients and had a larger number of cases. In addition, we try to combine serum albumin with the traditional prediction model to discuss its role in improving the scoring system. Chen $e t$ al. used the Immunome ${ }^{\mathrm{TM}}$ protein array system to explore protein expression in the plasma of IE patients. It was found that glyceraldehyde-3-phosphate dehydrogenase (GAPDH) and drebrin-like protein (DBNL) particularly may serve as valuable diagnostic biomarkers (25). For further research, the Immunome ${ }^{\mathrm{TM}}$ protein array system is a good choice to explore more potential biomarkers of proteomics for the diagnosis and prognosis of IE.

The results of this study suggest that preoperative serum albumin is a potential marker for predicting early postoperative mortality, which could increase the prediction of traditional models and help stratify risk. Serum albumin detection is a simple and inexpensive routine laboratory test for clinical use, and hypoalbuminemia is a reminder that patients should be targeted for treatment. In terms of the abundant physiological functions of albumin itself, supplementing albumin exogenously would appear to be the easiest solution. In different studies, the use of exogenous albumin remains a controversial method, and there is a lack of high-quality data to support its role (26). A large retrospective database study of 19,578 patients undergoing coronary artery bypass graft surgery showed that the use of albumin reduced all-cause mortality compared to nonprotein colloids (27). However, various recent retrospective studies have found no difference in major outcomes among cardiac surgery patients using colloidal or crystalline preparations $(28,29)$. In patients with sepsis and/or septic shock, research has shown that supplements of albumin might provide some improvement in hemodynamics, but do not affect short-term or long-term survival (30).

At present, many scholars believe that hypoalbuminemia is the result of a pathological state and should not be regarded as a disease requiring a specific treatment. In clinical practice, rather than simply supplying patients with exogenous albumin, the underlying cause of hypoalbuminemia should be treated or prevented. First, we need to evaluate and consider more comprehensive infection testing and antibiotic adjustments. Hypoproteinemia should alert clinicians to the possibility that IE patients have a severe infection, which is an important risk factor for death after heart surgery (31). Second, we also need to re-examine the nutritional status of patients and provide nutritional support as necessary. In a multicenter prospective study, poor nutritional status was found to be independently associated with an increased risk of death in elderly patients with IE (15). Finally, hypoproteinemia suggests that we also need to consider whether a patient has renal insufficiency. In addition to the disease progression of IE itself, which damages renal function (14), systemic inflammatory reaction and low perfusion caused by cardiopulmonary bypass surgery also cause great damage to renal function. Acute kidney injury is associated with high morbidity and mortality; thus, it is essential to prevent the occurrence of renal insufficiency $(14,32,33)$.

Although preoperative evaluation is important, postoperative management of patients should not be neglected. The 2015 ESC Guidelines for the management of infective endocarditis state that postoperative management of IE patients can follow the usual recommendations after valve replacement, but also take into account the specificity of IE (2). Postoperative management should be particularly careful because of the significantly increased risk of in-hospital mortality and postoperative complications in IE patients undergoing emergency or urgent surgery. Major complications include severe coagulopathy, re-exploration of the chest for bleeding, acute renal failure requiring hemodialysis, stroke, low cardiac output syndrome, pneumonia, atrioventricular block, etc. Studies have shown that the hospital mortality after surgery mainly depends on the clinical evolution after surgery. Renal failure and septic shock after surgery are serious risk factors for death (34). Therefore, patients must undergo a thorough clinical evaluation after surgery to achieve a good prognosis.

The current study had some limitations. First, the study was limited by the bias inherent in its retrospective design. Second, the sample size of this study was not very large. Finally, we defined a patient's albumin level at admission as the preoperative albumin level; however, patients' albumin levels will vary with treatment during preoperative preparations. This may have resulted in a discrepancy 
between albumin levels at admission and realistic albumin levels before surgery.

\section{Conclusions}

The results of this study suggest that preoperative serum albumin is inversely associated with early mortality after IE surgery, and is a promising prognostic indicator in preoperative risk stratification assessments of IE patients.

\section{Acknowledgments}

Funding: This work was supported by the National Natural Science Foundation of China (Grant numbers 81770319, 82070297 and 81570039) and the Natural Science Funds of Guangdong Province (Grant number 2019A1515010218).

\section{Footnote}

Reporting Checklist: The authors have completed the STARD reporting checklist. Available at https://dx.doi. org/10.21037/atm-21-3913

Data Sharing Statement: Available at https://dx.doi. org/10.21037/atm-21-3913

Conflicts of Interest: All authors have completed the ICMJE uniform disclosure form (available at https://dx.doi. org/10.21037/atm-21-3913). The authors have no conflicts of interest to declare.

Ethical Statement: The authors are accountable for all aspects of the work, including ensuring that any questions related to the accuracy or integrity of any part of the work have been appropriately investigated and resolved. The study was conducted according to the Declaration of Helsinki (as revised in 2013) and was approved by the institutional review board of The First Affiliated Hospital of Sun Yat-sen University (IRB-FAH-SYSU). Individual consent for this retrospective analysis was waived.

Open Access Statement: This is an Open Access article distributed in accordance with the Creative Commons Attribution-NonCommercial-NoDerivs 4.0 International License (CC BY-NC-ND 4.0), which permits the noncommercial replication and distribution of the article with the strict proviso that no changes or edits are made and the original work is properly cited (including links to both the formal publication through the relevant DOI and the license). See: https://creativecommons.org/licenses/by-nc-nd/4.0/.

\section{References}

1. Baddour LM, Wilson WR, Bayer AS, et al. Infective Endocarditis in Adults: Diagnosis, Antimicrobial Therapy, and Management of Complications: A Scientific Statement for Healthcare Professionals From the American Heart Association. Circulation 2015;132:1435-86.

2. Habib G, Lancellotti P, Antunes MJ, et al. 2015 ESC Guidelines for the management of infective endocarditis: The Task Force for the Management of Infective Endocarditis of the European Society of Cardiology (ESC). Endorsed by: European Association for Cardio-Thoracic Surgery (EACTS), the European Association of Nuclear Medicine (EANM). Eur Heart J 2015;36:3075-128.

3. Murdoch DR, Corey GR, Hoen B, et al. Clinical presentation, etiology, and outcome of infective endocarditis in the 21st century: the International Collaboration on Endocarditis-Prospective Cohort Study. Arch Intern Med 2009;169:463-73.

4. Anantha Narayanan M, Mahfood Haddad T, Kalil AC, et al. Early versus late surgical intervention or medical management for infective endocarditis: a systematic review and meta-analysis. Heart 2016;102:950-7.

5. Varela Barca L, Navas Elorza E, Fernández-Hidalgo $\mathrm{N}$, et al. Prognostic factors of mortality after surgery in infective endocarditis: systematic review and meta-analysis. Infection 2019;47:879-95.

6. Cahill TJ, Baddour LM, Habib G, et al. Challenges in Infective Endocarditis. J Am Coll Cardiol 2017;69:325-44.

7. Arques $\mathrm{S}$. Human serum albumin in cardiovascular diseases. Eur J Intern Med 2018;52:8-12.

8. Weimann A, Braga M, Carli F, et al. ESPEN guideline: Clinical nutrition in surgery. Clin Nutr 2017;36:623-50.

9. Karas PL, Goh SL, Dhital K. Is low serum albumin associated with postoperative complications in patients undergoing cardiac surgery? Interact Cardiovasc Thorac Surg 2015;21:777-86.

10. Kapoor PM, Narula J, Chowdhury UK, et al. Serum albumin perturbations in cyanotics after cardiac surgery: Patterns and predictions. Ann Card Anaesth 2016;19:300-5.

11. Yang F, Zhang B, Yu J, et al. Epidemiology and the prognosis of healthcare-associated infective endocarditis in China: the significance of non-nosocomial acquisition. Emerg Microbes Infect 2015;4:e38.

12. Krajinovic V, Ivancic $S$, Gezman $P$, et al. Association 
Between Cardiac Surgery and Mortality Among Patients With Infective Endocarditis Complicated by Sepsis and Septic Shock. Shock 2018;49:536-42.

13. Tamura K, Arai H, Yoshizaki T. Long-term outcome of active infective endocarditis with renal insufficiency in cardiac surgery. Ann Thorac Cardiovasc Surg 2012;18:216-21.

14. Von Tokarski F, Lemaignen A, Portais A, et al. Risk factors and outcomes of early acute kidney injury in infective endocarditis: A retrospective cohort study. Int J Infect Dis 2020;99:421-7.

15. Forestier E, Roubaud-Baudron C, Fraisse T, et al. Comprehensive geriatric assessment in older patients suffering from infective endocarditis. A prospective multicentric cohort study. Clin Microbiol Infect 2019;25:1246-52.

16. Arques $S$, Ambrosi P. Human serum albumin in the clinical syndrome of heart failure. J Card Fail 2011;17:451-8.

17. Kang DH. Timing of surgery in infective endocarditis. Heart 2015;101:1786-91.

18. Ballmer PE. Causes and mechanisms of hypoalbuminaemia. Clin Nutr 2001;20:271-3.

19. Hotamisligil GS. Inflammation and metabolic disorders. Nature 2006;444:860-7.

20. Le V, Gill S. Serious complications after infective endocarditis. Dan Med Bull 2010;57:A4192.

21. Kremer H, Baron-Menguy C, Tesse A, et al. Human serum albumin improves endothelial dysfunction and survival during experimental endotoxemia: concentrationdependent properties. Crit Care Med 2011;39:1414-22.

22. van Beek DEC, van der Horst ICC, de Geus AF, et al. Albumin, a marker for post-operative myocardial damage in cardiac surgery. J Crit Care 2018;47:55-60.

23. Kahraman S, Zencirkıran Aguş H, Kalkan AK, et al. Prognostic nutritional index predicts mortality in infective endocarditis. Turk Kardiyol Dern Ars 2020;48:392-402.

24. Siciliano RF, Gualandro DM, Bittencourt MS, et al. Biomarkers for prediction of mortality in left-sided infective endocarditis. Int J Infect Dis 2020;96:25-30.

Cite this article as: Huang S, Zhou Z, Luo L, Yue Y, Liu Q, Feng K, Hou J, Wang K, Chen J, Li H, Huang L, Fu G, Chen G, Liang M, Wu Z. Preoperative serum albumin: a promising indicator of early mortality after surgery for infective endocarditis. Ann Transl Med 2021;9(18):1445. doi: 10.21037/ atm-21-3913
25. Chen $\mathrm{CH}$, Chen YC, Huang CH, et al. Exploring Potential Proteomic Biomarkers for Prognosis of Infective Endocarditis through Profiled Autoantibodies by an Immunomics Protein Array Technique. Heart Surg Forum 2020;23:E555-73.

26. Hanley C, Callum J, Karkouti K, et al. Albumin in adult cardiac surgery: a narrative review. Can J Anaesth 2021;68:1197-213.

27. Sedrakyan A, Gondek K, Paltiel D, et al. Volume expansion with albumin decreases mortality after coronary artery bypass graft surgery. Chest 2003;123:1853-7.

28. Rabin J, Meyenburg T, Lowery AV, et al. Restricted Albumin Utilization Is Safe and Cost Effective in a Cardiac Surgery Intensive Care Unit. Ann Thorac Surg 2017; 104:42-8.

29. Fink RJ, Young A, Yanez ND, et al. Cohort Study of Albumin versus Lactated Ringer's for Postoperative Cardiac Surgery Fluid Resuscitation in the Intensive Care Unit. Pharmacotherapy 2018;38:1241-9.

30. Caironi P, Tognoni G, Masson S, et al. Albumin replacement in patients with severe sepsis or septic shock. N Engl J Med 2014;370:1412-21.

31. Rapp-Kesek D, Ståhle E, Karlsson TT. Body mass index and albumin in the preoperative evaluation of cardiac surgery patients. Clin Nutr 2004;23:1398-404.

32. Ortiz-Soriano V, Donaldson K, Du G, et al. Incidence and Cost of Acute Kidney Injury in Hospitalized Patients with Infective Endocarditis. J Clin Med 2019;8:927.

33. Guo M, St Pierre E, Clemence J Jr, et al. Impact of Chronic Renal Failure on Surgical Outcomes in Patients With Infective Endocarditis. Ann Thorac Surg 2021;111:828-35.

34. García-Granja PE, López J, Vilacosta I, et al. Surgery changes prognosis in infective endocarditis: The importance of post-surgical clinical evolution. Eur J Intern Med 2018;55:52-6.

(English Language Editor: L. Huleatt) 


\section{Supplementary}

Table S1 Univariate and multivariate logistic regression analyses showing the association between preoperative hypoalbuminemia (albumin $<3.5 \mathrm{~g} / \mathrm{dL}$ ) and the risk of early mortality in surgically treated infective endocarditis patients

\begin{tabular}{lll}
\hline & Odds ratio $(95 \% \mathrm{Cl})$ & $\mathrm{P}$ value \\
\hline $\begin{array}{l}\text { Univariate analysis } \\
\text { Hypoalbuminemia }\end{array}$ & $3.95(1.47-10.64)$ & 0.006 \\
Multivariate analysis & & \\
Model 1 & & \\
Hypoalbuminemia & $4.06(1.50-10.97)$ & 0.006 \\
EurosCORE II & $0.95(0.76-1.19)$ & 0.643 \\
Model 2 & & \\
Hypoalbuminemia & $3.37(1.23-9.24)$ & 0.018 \\
Charlson score & $1.48(1.13-1.94)$ & 0.005 \\
Model 3 & & \\
Hypoalbuminemia & $3.09(1.02-9.35)$ & 0.046 \\
Age, per year & $1.01(0.98-1.04)$ & 0.650 \\
Heart failure & $8.21(2.96-22.81)$ & $<0.001$ \\
Renal insufficiency & $2.20(0.46-10.46)$ & 0.323 \\
Multiple valve infection & $3.86(1.31-11.41)$ & 0.015 \\
Vegetation size, per 1 mm & $0.99(0.92-1.06)$ & 0.689 \\
\hline
\end{tabular}

EuroSCORE, European System for Cardiac Operative Risk Evaluation.
Table S2 Preoperative hypoalbuminemia in relation to prediction of early mortality in IE.

\begin{tabular}{lccc}
\hline \multirow{2}{*}{$\begin{array}{l}\text { Preoperative } \\
\text { hypoalbuminemia }\end{array}$} & \multicolumn{2}{c}{ Early mortality } & Total \\
\cline { 2 - 3 } & Positive & Negative & \\
\hline Positive & 14 & 95 & 109 \\
Negative & 6 & 161 & 167 \\
Total & 20 & 256 & 276 \\
\hline
\end{tabular}

